\title{
The disjoint arcs property for homogeneous curves
}

\author{
by \\ Paweł Kr upski (Wrocław)
}

\begin{abstract}
The local structure of homogeneous continua (curves) is studied. Components of open subsets of each homogeneous curve which is not a solenoid have the disjoint arcs property. If the curve is aposyndetic, then the components are nonplanar. A new characterization of solenoids is formulated: a continuum is a solenoid if and only if it is homogeneous, contains no terminal nontrivial subcontinua and small subcontinua are not $\infty$-ods.
\end{abstract}

0. Introduction. All spaces in the paper are metric separable and all maps are continuous. A space $X$ is homogeneous if for each two points $x, y \in$ $X$ there exists a homeomorphism $h: X \rightarrow X$ such that $h(x)=y$. A curve is a one-dimensional continuum. A space $X$ has the disjoint arcs property $(D A P)$ if any two paths in $X$ can be approximated, arbitrarily closely, by disjoint paths. More precisely, $X$ has the DAP if for each $\varepsilon>0$ and for any two maps $f, g: I=[0,1] \rightarrow X$ there exist maps $f^{\prime}, g^{\prime}: I \rightarrow X$ such that $f^{\prime}(I) \cap g^{\prime}(I)=\emptyset$ and $\widehat{\varrho}\left(f, f^{\prime}\right)<\varepsilon, \widehat{\varrho}\left(g, g^{\prime}\right)<\varepsilon$, where $\widehat{\varrho}$ denotes the sup-norm metric induced by $\varrho$ in $X$. If, in the definition, $I$ is replaced by the $n$ dimensional disk, one gets the disjoint $n$-disks property $\left(D D^{n} P\right)$. The latter property turned out to be crucial in recognizing $n$-dimensional Menger space manifolds among $\mathrm{LC}^{n-1}$-spaces [2]. The $\mathrm{DD}^{n} \mathrm{P}$ is a kind of a general position property, so it requires enough room - for manifolds this is guaranteed by the sufficiently high dimension, whereas Menger space manifolds have a special structure which is responsible for the $\mathrm{DD}^{n} \mathrm{P}$.

In [8] it was observed that all homogeneous locally compact locally connected spaces of dimension at least three have the DAP. Dimension two is not friendly to the DAP because no two-dimensional manifold has the property.

1991 Mathematics Subject Classification: 54F50, 54F65.

Key words and phrases: homogeneous continuum, aposyndetic curve, solenoid, disjoint arcs property, Menger universal curve. 
In this paper we prove that components of open subsets of a homogeneous curve have the DAP unless the curve is a solenoid. In particular, all homogeneous curves which are not simple closed curves have the DAP. To get this we first investigate the local structure of homogeneous continua. Roughly speaking, if a homogeneous curve $X$ is neither the Menger universal curve nor a solenoid, then either its small open subsets have nowhere dense components which are Menger curve manifolds or each component $C$ of an arbitrary open subset of $X$ is covered by open subsets of $C$ that split into components nowhere dense in $C$.

We also show that if a homogeneous curve $X$ is not a simple closed curve but is aposyndetic, then components of open subsets of $X$ are nonplanar. A new characterization of solenoids is formulated (Theorem 3.1) which improves others of that sort.

By a solenoid we mean a space homeomorphic to the inverse limit of a sequence of circles with covering bonding maps. We say that small subcontinua of a continuum $X$ have property $\mathcal{P}$ if there is a positive number $\alpha$ such that each subcontinuum of $X$ of diameter less than $\alpha$ satisfies $\mathcal{P}$. A space $X$ is locally connected at a point $x$ if any open neighborhood of $x$ contains a connected neighborhood of $x$.

If $X$ is a space with metric $\varrho$, then a homeomorphism $h: X \rightarrow X$ is called an $\varepsilon$-homeomorphism if $\varrho(z, h(z))<\varepsilon$ for each $z \in X$. We will use the following theorem of Effros [5].

Proposition 0.1. Suppose $X$ is a homogeneous compact space with metric $\varrho$. Then for every $\varepsilon>0$ there exists $\delta>0$ such that if $\varrho(x, y)<\delta$, then there is an $\varepsilon$-homeomorphism $h: X \rightarrow X$ such that $h(x)=y$ (the number $\delta$ is called an Effros $\delta$ for $\varepsilon$ ).

The author is very indebted to K. Omiljanowski and J. Prajs for their criticism and valuable suggestions.

1. Menger curve manifolds. Recall that the Menger universal curve is characterized as a locally connected curve with no local separating points and containing no planar open nonempty subset [1] $(x \in X$ is a local separating point of $X$ if $U-\{x\}$ is not connected for some connected open subset $U$ of $X$ ). A space is said to be a Menger curve manifold if it can be covered by open subsets homeomorphic to open subsets of the Menger universal curve.

The Sierpiński universal planar curve is homeomorphic to any locally connected planar curve with no local separating points [22].

A space $X$ is locally homogeneous if for any two points $x, y \in X$ there exist open neighborhoods $U$ of $x$ and $V$ of $y$ and a homeomorphism $h: U \rightarrow$ $V$ such that $h(x)=y$. It is known that the Sierpiński universal planar curve 
and any of its open nonempty subsets are not locally homogeneous (each homeomorphism maps points of the boundary of a complementary domain onto points of the boundary of another complementary domain of the curve $[7])$.

The following lemma is implicit in [1] but, for the reader's convenience, we include a proof.

Lemma 1.1. Suppose $X$ is a one-dimensional locally compact locally connected space with no local separating points. Then either $X$ is a Menger curve manifold or $X$ contains an open nonempty subset homeomorphic to an open subset of the Sierpinski universal planar curve.

Proof. By [21, (15.43), p. 22] each point $x \in X$ is contained in an arbitrarily small connected open subset $R$ of $X$ having property $S$. Since cl $R$ is a locally connected continuum $[21,(15.3)$, p. 20$]$, it admits a decreasing sequence of order two brick partitions [12, Theorem 2.9]. The brick partitions provide us with a connected and uniformly locally connected open neighborhood $G$ of $x$, contained in $R$. Indeed, if there is an element $U$ of a brick partition $\mathcal{U}$ such that $x \in \operatorname{int} U \subset R$, then $G=U$; otherwise $x$ is contained in the interior $G$ of the union of two elements of $\mathcal{U}$. In either case $G$ is uniformly locally connected. We infer from [12, Proposition 2.5] that $\mathrm{cl} G$ is a locally connected curve such that each point of bd $G$ is non-locally-separating in $\operatorname{cl} G$. That no point of $G$ locally separates $\operatorname{cl} G$ follows from the hypothesis and from [12, Proposition 2.4]. Thus $\mathrm{cl} G$ is a locally connected curve without local separating points. Now, if no nonempty open set in $X$ is planar, then $\mathrm{cl} G$ is homeomorphic to the Menger universal curve and $X$ is a Menger curve manifold. Otherwise we can assume that $\operatorname{cl} G$ is planar but then $\operatorname{cl} G$ is homeomorphic to the Sierpiński universal planar curve.

LEMMA 1.2. Let $X$ be a nondegenerate homogeneous continuum with metric $\varrho$ and let $C$ be a component of an open proper subset $U$ of $X$. Suppose $c \in C$ and $0<\varepsilon<\frac{1}{7} \varrho(c, X-U)$. If $W$ is a connected open (in $C$ ) neighborhood of $c$ of diameter less than $\varepsilon$, then for any $\varepsilon$-homeomorphism $h: X \rightarrow X$ such that $h(W) \cap W \neq \emptyset$ the set $h(W)$ is an open subset of $C$.

P r o o f. Obviously $h(W) \subset C$. Suppose $h(W)$ is not open in $C$, i.e., there exist a point $d \in h(W)$ and a sequence of points $d_{n} \in C-h(W)$ converging to $d$. Let $\alpha=\min \{\varrho(c, C-W), \varepsilon\}$ and define $V=\bigcup\{g(W \cup h(W)): g$ is an $\alpha$-homeomorphism of $X$ onto $X\}$. It follows from Proposition 0.1 that $V$ is open in $X$, so the set $V^{\prime}=V \cap C$ is open in $C$ and contains $d$. Moreover, $V^{\prime}$ is connected, because of the choice of $\varepsilon$ and $\alpha$, and its diameter is less than $5 \varepsilon$. The set $h^{-1}\left(V^{\prime}\right)$ is connected of diameter less than $7 \varepsilon$ and contains $c$, hence it is contained in $C$. We can assume that $d_{n} \in V^{\prime}$ for each $n$, so 
$h^{-1}\left(d_{n}\right) \in C$. On the other hand, $h^{-1}\left(d_{n}\right)$ converges to $h^{-1}(d) \in W$, thus $h^{-1}\left(d_{n}\right) \in W$ and $d_{n} \in h(W)$ for large $n$, a contradiction.

LEMma 1.3. Let $X$ be a nondegenerate homogeneous continuum with metric $\varrho$ and let $C$ be a component of a nonempty open subset $U$ of $X$. Then the set of points of $C$ at which $C$ is locally connected is open in $C$. If, moreover, $X$ is not a solenoid, then the set is contained in the set of points of $C$ which do not locally separate $C$.

Remark. Being a local separating point of a space and of its open subset is the same if the space is locally connected at that point.

Proof of $\mathrm{Lemma}$ 1.3. The conclusion is obvious if $U=X$. Assume $C$ is locally connected at $c \in C$ and $U \neq X$. For $0<\varepsilon<\frac{1}{7} \varrho(c, X-U)$ take an Effros $\delta$ less than $\varepsilon$. Let $W$ be a connected, open (in $C$ ) neighborhood of $c$ of diameter less than $\delta$. Consider an arbitrary point $x \in W$. By Proposition 0.1 there exists an $\varepsilon$-homeomorphism $h: X \rightarrow X$ such that $h(c)=x$. It follows from Lemma 1.2 that $h(W)$ is a connected, open neighborhood of $x$ in $C$. Therefore $C$ is locally connected at $x$ and this gives the first part of the conclusion.

Suppose now that $c$ locally separates $C$. Without loss of generality, we can assume that $W-\{c\}$ is not connected. Then $h(W)-\{x\}$ is not connected, which means that each point of $W$ locally separates $W$ (see the remark preceding the proof). Observe that $W$, being an open subset of the component $C$ of $U$, is locally compact. By [21, (9.2), p. 61], $W$ contains a dense subset of points of order 2. Proposition 0.1 and Lemma 1.2 imply that each point of $W$ has order 2. Now, it easily follows from the arcwise connectedness of $W$ that $W$ is an arc without end-points. The homogeneity of $X$ yields that small subcontinua of $X$ are arcs, but then $X$ is a solenoid [15], a contradiction.

We do not know whether the set of points of local connectedness of $C$ is closed in $C$.

One can meet, a priori, two kinds of points of non-local connectedness in a component $C$ of an open subset $U$ of a homogeneous continuum. Sufficiently small neighborhoods in $C$ of points of the first kind have uncountably many components nowhere dense in $C$. Other points of non-local connectedness are of the second kind.

The next lemma says that any shrinking of $U$ converts points of the second kind into points of local connectedness.

Lemma 1.4. Let $X$ be a homogeneous continuum and let $C$ be a component of an open subset $U$ of $X$. Suppose $c \in C$ and there is a sequence of subcontinua $K_{n}$ of $C$, with nonempty interiors relative to $C$, such that 
$K_{n} \rightarrow\{c\}$ as $n \rightarrow \infty$. If $C^{\prime}$ is the component of $c$ in an open subset $U^{\prime}$ of $X$ such that $U^{\prime} \subset \operatorname{cl} U^{\prime} \subset U$, then $C^{\prime}$ is locally connected at $c$.

Proof. For each $n$ choose a point $c_{n} \in \operatorname{int}_{C} K_{n}$. By Proposition 0.1 there exists a sequence of homeomorphisms $h_{n}: X \rightarrow X$, uniformly converging to the identity map, such that $h_{n}\left(c_{n}\right)=c$. We can assume that $h_{n}\left(K_{n}\right) \subset C^{\prime}$ and $h_{n}^{-1}\left(C^{\prime}\right) \subset C$ for each $n$. Suppose $C^{\prime}$ is not locally connected at $c$. This means that there exists $m$ such that $c \notin \operatorname{int}_{C^{\prime}} h_{n}\left(K_{n}\right)$ for $n>m$. Fix $n>m$. There is a sequence of points $d_{k} \in C^{\prime}$ such that $d_{k} \rightarrow c$ as $k \rightarrow \infty$, and $d_{k} \notin h_{n}\left(K_{n}\right)$ for each $k$. Then $h_{n}^{-1}\left(d_{k}\right) \rightarrow c_{n}$ as $k \rightarrow \infty$, so $h_{n}^{-1}\left(d_{k}\right) \notin C$ for large $k$. But, on the other hand, $h_{n}^{-1}\left(d_{k}\right) \in h_{n}^{-1}\left(C^{\prime}\right) \subset C$, a contradiction.

Shrinking open subsets of a space does not change local connectedness of their components. More precisely, we have the following obvious lemma.

LEMma 1.5. If a component $C$ of an open subset $U$ of a space $X$ is locally connected at $c \in C$ and $C^{\prime}$ is the component of $c$ in an open subset $U^{\prime} \subset U$, then $C^{\prime}$ is locally connected at $c$.

Lemma 1.6. Let $X$ be a non-locally-connected homogeneous continuum. Suppose some component of a certain open subset of $X$ is locally connected at some point. Then there exists a number $\alpha>0$ such that each nonempty open subset of $X$ of diameter less than $\alpha$ consists of uncountably many nowhere dense and locally connected components.

Proof. Let $C$ be a component of an open subset $U \subset X$ and suppose $C$ is locally connected at $c \in C$. First, we find an open neighborhood $V_{c}$ of $c$ with all components locally connected and nowhere dense. It follows from Lemma 1.3 that there exists an arbitrarily small connected and locally connected subset $W$ of $C$ which is open in $C$ and contains $c$. The set is of the form $W=C \cap V$, where $V$ is some open subset of $U$. Obviously, $W$ is a locally connected component of $V$ but one cannot say that about other components of $V$. To get the desired neighborhood $V_{c}$ we, therefore, appropriately shrink $V$. Namely, by Lemma 1.5 and Proposition 0.1, there exist two sets $W_{c}$ and $V_{c}$ satisfying the following conditions:

(1) $c \in W_{c}$

(2) $W_{c}$ is connected, locally connected and relatively open in $C$,

(3) $W_{c}=V_{c} \cap C$,

(4) $V_{c}$ is an open subset of $V$,

(5) for any $x \in V_{c}$ there is a homeomorphism $h: X \rightarrow X$ such that $h(c)=x$ and $h(V) \supset V_{c}$.

Since $h(W)$ is a locally connected component of $h(V)$, Lemma 1.5 and condition (5) imply that each component of $V_{c}$ is locally connected. Notice 
that $V_{c}$ can be considered arbitrarily small. That components of $V_{c}$ are nowhere dense now follows from the hypothesis and the homogeneity. The Baire Category Theorem applied to $V_{c}$ assures that $V_{c}$ has uncountably many components.

By the homogeneity, every point $x \in X$ has such a neighborhood $V_{x}$. Let $\alpha$ be the Lebesgue number for the cover $\left\{V_{x}\right\}$ of $X$. Lemma 1.5 implies that all components of each nonempty open subset $A$ of $X$ with diam $A<\alpha$ are locally connected. Evidently, components of $A$ are nowhere dense and their number is uncountable by the Baire Category Theorem.

THEOREM 1.7. If $X$ is a homogeneous continuum, then one of the following three cases holds.

(1) $X$ is locally connected or a solenoid.

(2) There exists an $\alpha>0$ such that each open subset of $X$ of diameter less than $\alpha$ consists of uncountably many nowhere dense components which are locally connected and have no local separating points.

(3) Each component $C$ of an arbitrary open subset of $X$ has an open cover by subsets of $C$ consisting of uncountably many components nowhere dense in $C$.

Pr o o f. Suppose neither (1) nor (3) is satisfied. Then there exist an open subset $U$ of $X$, its component $C$, a point $c \in C$ and a sequence of subcontinua $K_{n} \subset C$ with nonempty interiors relative to $C$ such that $K_{n} \rightarrow\{c\}$ as $n \rightarrow \infty$. By Lemma 1.4 the component $C^{\prime}$ of some open set $U^{\prime} \subset U$ such that $c \in C^{\prime}$ is locally connected at $c$. To complete the proof apply Lemmas 1.6 and 1.3 .

THEOREM 1.8. If $X$ is a homogeneous curve, then one of the following three cases holds.

(1) $X$ is the Menger universal curve or a solenoid.

(2) There exists an $\alpha>0$ such that each open subset of $X$ of diameter less than $\alpha$ consists of uncountably many nowhere dense components which are Menger curve manifolds.

(3) Case (3) of Theorem 1.7.

Pro of. Suppose neither (1) nor (3) holds. Then, by [1], $X$ is not locally connected and case (2) of Theorem 1.7 holds. Let $C$ be a component of an open subset $U$ of $X$ with $\operatorname{diam} U<\alpha$. Suppose $C$ is not a Menger curve manifold. By Lemma 1.1, $C$ contains an open (in $C$ ) connected subset $W$ homeomorphic to an open subset of the Sierpiński universal planar curve. Let $\varepsilon$ be as in Lemma 1.2. Take an Effros $\delta$ for $\varepsilon$. We can assume that diam $W<$ $\delta$. Now, it easily follows from Lemma 1.2 that $W$ is locally homogeneous, which is impossible for an open subset of the Sierpiński curve. 


\section{The disjoint arcs property}

THEOREM 2.1. If $X$ is a homogeneous curve which is not a solenoid, then each component of an arbitrary open subset of $X$ has the DAP. In particular, each homogeneous curve which is not a simple closed curve has the DAP.

Pr o of. Since the Menger universal curve satisfies the assertion (see [2]), it remains to consider cases (2) and (3) of Theorem 1.8.

Denote by $\varrho$ a metric in $X$. Let $C$ be a component of an open subset $U$ of $X$ and suppose two maps $f$ and $g$ of $I=[0,1]$ into $C$ are given such that $f(I) \cap g(I) \neq \emptyset$. Set $K=f(I) \cup g(I)$ and $\eta=\varrho(K, X-U)$.

Assume case (2) holds. Let $\mathcal{U}$ be a finite open cover of $X$ with mesh $\mathcal{U}<$ $\min \{\alpha, \eta\}$. Partition $I$ into smaller intervals $I_{i}=\left[t_{i-1}, t_{i}\right]$ such that $0=$ $t_{0}<t_{1}<\ldots<t_{n}=1$ and, for every $i=1, \ldots, n, f\left(I_{i}\right) \subset U_{i}$ and $g\left(I_{i}\right) \subset V_{i}$ for some $U_{i}, V_{i} \in \mathcal{U}$. Denote by $A_{i}$ and $B_{i}$ the components of $U_{i}$ and $V_{i}$ containing $f\left(I_{i}\right)$ and $g\left(I_{i}\right)$, respectively. Put $M=\bigcup_{i=1}^{n}\left(A_{i} \cup B_{i}\right)$. Observe that if $D$ is a component of a set of the form $A_{i} \cap A_{j}, A_{i} \cap B_{j}$ or $B_{i} \cap B_{j}$, then $D$ is a Menger curve manifold. This follows from the fact that $D$ coincides with a component of $U_{i} \cap U_{j}, U_{i} \cap V_{j}$ or $V_{i} \cap V_{j}$, respectively, and from condition (2). Consequently, $M$ is a Menger curve manifold (see Lemma 1.1). Since $K \subset M$ and $M$ has the DAP [2], the maps $f$ and $g$ are approximated arbitrarily closely by maps $f^{\prime}$ and $g^{\prime}$ of $I$ into $M \subset C$ with disjoint images.

Assume now case (3) holds. Let $\mathcal{W}$ be a cover of $C$ by open subsets of $C$ consisting of uncountably many components nowhere dense in $C$. Partition $I$ into intervals $I_{i}=\left[t_{i-1}, t_{i}\right], 0=t_{0}<t_{1}<\ldots<t_{n}=1$, such that, for every $i=1, \ldots, n, f\left(I_{i}\right) \subset W_{i}$ for some $W_{i} \in \mathcal{W}$.

We will construct, for every $\varepsilon>0$, an $\varepsilon$-approximation $f^{\varepsilon}: I \rightarrow C$ of $f$ such that $f^{\varepsilon}(I) \cap g(I)=\emptyset$. To this end, we will define by induction, for every $\varepsilon>0$ and $k=1, \ldots, n$, a partial $\varepsilon$-approximation $f_{k}^{\varepsilon}: I_{1} \cup \ldots \cup I_{k} \rightarrow C$ such that $f_{k}^{\varepsilon}\left(I_{1} \cup \ldots \cup I_{k}\right) \cap g(I)=\emptyset$ and then put $f^{\varepsilon}=f_{n}^{\varepsilon}$.

Proposition 0.1 will be repeatedly used in the construction without explicit mention but we hope such places can be readily recognized.

If $f\left(I_{1}\right) \cap g(I)=\emptyset$, then put $f_{1}^{\varepsilon}=f \mid I_{1}$ for every $\varepsilon>0$. Suppose

$$
f\left(I_{1}\right) \cap g(I) \neq \emptyset .
$$

Let $0<\varepsilon<\varrho\left(f\left(I_{1}\right), C-W_{1}\right)$ and take an Effros $\delta$ for $\varepsilon$. Denote by $D$ the component of $W_{1}$ containing $f\left(I_{1}\right)$. Since $W_{1}$ consists of uncountably many components which are nowhere dense in $C$ and $g(I)$ is locally connected, there is a component $E$ of $W_{1}$ different from $D$ such that $E \cap g(I)=\emptyset$ and $\varrho\left(f\left(I_{1}\right), E\right)<\delta$. Take an $\varepsilon$-homeomorphism $h: X \rightarrow X$ such that $h f\left(I_{1}\right) \subset E$ and put $f_{1}^{\varepsilon}=h f \mid I_{1}$. Thus the first inductive step is done. 
Suppose further that an $\varepsilon$-aproximation $f_{k}^{\varepsilon}: I_{1} \cup \ldots \cup I_{k} \rightarrow C$ of $f \mid I_{1} \cup$ $\ldots \cup I_{k}$ has been defined for every $\varepsilon>0$ such that $f_{k}^{\varepsilon}\left(I_{1} \cup \ldots \cup I_{k}\right) \cap g(I)=\emptyset$. Again, there are two cases to be considered. Assume first $f\left(I_{k+1}\right) \cap g(I)=\emptyset$. Let $0<\varepsilon<\min \left\{\eta, \varrho\left(f\left(I_{k+1}\right), g(I)\right)\right\}$. Take an Effros $\delta$ for $\varepsilon$ and an $\varepsilon$ homeomorphism $h: X \rightarrow X$ such that $h f\left(t_{k}\right)=f_{k}^{\delta}\left(t_{k}\right)$. Define $f_{k+1}^{\varepsilon}$ : $I_{1} \cup \ldots \cup I_{k+1} \rightarrow C$ by $f_{k+1}^{\varepsilon} \mid I_{1} \cup \ldots \cup I_{k}=f_{k}^{\delta}$ and $f_{k+1}^{\varepsilon}\left|I_{k+1}=h f\right| I_{k+1}$.

Assume now that $f\left(I_{k+1}\right) \cap g(I) \neq \emptyset$. Let $0<\varepsilon<\min \left\{\varrho\left(f\left(I_{k+1}\right), C-\right.\right.$ $\left.\left.W_{k+1}\right), \eta\right\}$ and let $\delta$ be an Effros $\delta$ for $\varepsilon / 2$. Define a map $F: I_{1} \cup \ldots \cup I_{k+1} \rightarrow$ $C$ by $F \mid I_{1} \cup \ldots \cup I_{k}=f_{k}^{\delta}$ and $F\left|I_{k+1}=h f\right| I_{k+1}$, where $h: X \rightarrow X$ is an $\varepsilon / 2$-homeomorphism such that $h f\left(t_{k}\right)=f_{k}^{\delta}\left(t_{k}\right)$. The map $F$ is an $\varepsilon / 2$ approximation of $f \mid I_{1} \cup \ldots \cup I_{k+1}$. If $F\left(I_{k+1}\right) \cap g(I)=\emptyset$, then let $f_{k+1}^{\varepsilon}=F$. In the case $F\left(I_{k+1}\right) \cap g(I) \neq \emptyset$ we proceed as in $(*)$, replacing $f$ and $I$ by $F$ and $I_{k+1}$. Namely, let $\delta^{\prime}$ be an Effros $\delta$ for $\varepsilon^{\prime}=\min \left\{\varrho\left(F\left(I_{1} \cup \ldots \cup I_{k}\right), g(I)\right), \delta\right\}$. We have $F\left(I_{k+1}\right) \subset W_{k+1}$ by construction. Denote by $D$ the component of $W_{k+1}$ containing $F\left(I_{k+1}\right)$ and find a component $E$ of $W_{k+1}$ different from $D$ such that $E \cap g(I)=\emptyset$ and $\varrho\left(F\left(I_{k+1}\right), E\right)<\delta^{\prime}$. Take an $\varepsilon^{\prime}$-homeomorphism $h^{\prime}: X \rightarrow X$ such that $h^{\prime} F\left(I_{k+1}\right) \subset E$ and define $f_{k+1}^{\varepsilon}=h^{\prime} F$. Then $f_{k+1}^{\varepsilon}$ maps $I_{1} \cup \ldots \cup I_{k+1}$ into $C, f_{k+1}^{\varepsilon}$ is $\varepsilon$-close to $f \mid I_{1} \cup \ldots \cup I_{k+1}$ and $f_{k+1}^{\varepsilon}\left(I_{1} \cup\right.$ $\left.\ldots \cup I_{k+1}\right) \cap g(I)=\emptyset$.

The induction is complete.

Theorem 2.1 can be viewed as yet another characterization of solenoids: a curve $X$ is a solenoid if and only if $X$ is homogeneous and some component of an open subset of $X$ does not have the DAP.

Remark. If each arcwise connected homogeneous curve were locally connected (this is an open problem), then we could get the DAP for homogeneous curves much easier. Namely, if a homogeneous curve $X$ contains no arc, then the DAP trivially holds. If $X$ contains an arc, then its arc components are dense [19]. So, if $X$ has at least two arc components, then, using Proposition 0.1, one immediately gets the DAP. If $X$ is arcwise connected and is not a simple closed curve, then, assuming the conjecture is true, $X$ is homeomorphic to the Menger universal curve (see [1]), which has the DAP.

3. A characterization of solenoids. A subcontinuum $A$ of a continuum $X$ is called terminal if, for any subcontinuum $B$ of $X$ intersecting $A$, either $A \subset B$ or $B \subset A$. Solenoids have no nontrivial terminal subcontinua because their proper nondegenerate subcontinua are arcs. The reader can find more about terminal subcontinua in the next section.

A continuum $X$ is a $\theta$-continuum if the complement of every subcontinuum of $X$ has a finite number of components. A continuum $X$ is called a hereditary $\theta$-continuum if each subcontinuum of $X$ is a $\theta$-continuum. By an $\infty$-od we mean a continuum containing a subcontinuum whose com- 
plement has infinitely many components. So, a continuum is a hereditary $\theta$-continuum if it contains no $\infty$-od. In what follows we prefer to use the " $\infty$-od" terminology.

The proof of Theorem 3.1 essentially relies on results of [10, Section 2]. One can easily check that statements $(2.1)-(2.3)$ of [10] remain true if the hypothesis that $X$ is a hereditary $\theta$-continuum is replaced by the condition that small subcontinua of $X$ are not $\infty$-ods, provided that one plays only with small subcontinua of $X$.

Theorem 3.1. A nondegenerate continuum $X$ is a solenoid if and only if $X$ is homogeneous, small subcontinua of $X$ are not $\infty$-ods and $X$ contains no proper nondegenerate terminal subcontinuum.

Proof. The necessity is obvious. Suppose $X$ satisfies the conditions. Then $[10,(2.3)]$, in a version for small subcontinua, says that

(i) small subcontinua of $X$ are decomposable;

(ii) small irreducible subcontinua of $X$ are of type $\lambda$;

(iii) the layers of small irreducible subcontinua of $X$ are degenerate.

Therefore small irreducible subcontinua of $X$ are arcs. Then, arguing as in the proof of $[10,(2.5)]$, one infers that small subcontinua of $X$ are atriodic. Finally, use [4, Theorem 2]: if a nondegenerate homogeneous continuum $X$ contains an arc and small subcontinua of $X$ are atriodic, then $X$ is a solenoid.

Theorem 3.1 improves the characterizations of [10, (3.8)], [11, (4.8)], [15] and [16], since nontrivial terminal subcontinua of homogeneous curves are hereditarily indecomposable (see the next section).

4. Aposyndetic curves. A space $X$ is said to be aposyndetic if for any two distinct points $x, y \in X$ there exists a continuum $K$ such that $x \in \operatorname{int} K \subset K \subset X-\{y\}$. Locally connected continua are aposyndetic but there are uncountably many, topologically different, aposyndetic homogeneous curves which are not locally connected (see [3], [17], [18], [13]). All of them are locally Cantor bundles of the Menger universal curves. On the other hand, relatively few general facts are known about such curves; for example, it is not known if they must contain an arc. The reason for studying aposyndetic homogeneous curves is that they naturally arise as quotient spaces of terminal decompositions of decomposable homogeneous curves. Namely, the Terminal Decomposition Theorem (see [20] or [9]) says that each homogeneous curve continuously decomposes into maximal terminal subcontinua which are homogeneous hereditarily indecomposable tree-like subcontinua and the quotient space of the decomposition is again a homo- 
geneous curve. In the case where the curve is decomposable the quotient space is also aposyndetic (this was first proved by Jones [6]).

The following proposition follows from the above remarks.

Proposition 4.1. If $X$ is a homogeneous curve, then $X$ is aposyndetic if and only if $X$ is decomposable and contains no nondegenerate proper terminal subcontinuum.

Proposition 4.1 and Theorem 3.1 imply that each homogeneous aposyndetic curve which is not a simple closed curve contains arbitrarily small $\infty$-ods. Actually, Proposition 0.1 and the homogeneity give something more.

Proposition 4.2. If $X$ is a homogeneous aposyndetic curve which is not a simple closed curve, then each point of $X$ belongs to arbitrarily small $\infty$-ods.

TheOREM 4.3. If $X$ is a homogeneous aposyndetic curve which is not a simple closed curve, then none of the components of an arbitrary open nonempty subset of $X$ is planar.

Pro of. Clearly $X$ is not a solenoid. The conclusion is evident if $X$ is the Menger universal curve. Suppose $C$ is a component of an open nonempty subset $U$ of $X$ and $X$ is not the Menger universal curve. We now use Theorem 1.8. If its case (2) holds, then $C$ contains a Menger curve manifold which is not planar. Case (3) combined with Proposition 4.2 implies that $C$ contains uncountably many disjoint $\infty$-ods, hence $C$ cannot be planar by the wellknown theorem of Moore [14].

\section{References}

[1] R. D. Anderson, One-dimensional continuous curves and a homogeneity theorem, Ann. of Math. 68 (1958), 1-16.

[2] M. Bestvina, Characterizing k-dimensional universal Menger compacta, Mem. Amer. Math. Soc. 380 (1988).

[3] J. H. Case, Another 1-dimensional homogeneous continuum which contains an arc, Pacific J. Math. 11 (1961), 455-469.

[4] E. Duda, P. Krupski and J. T. Rogers, On locally chainable homogeneous continua, Topology Appl. 42 (1991), 95-99.

[5] E. G. Effros, Transformation groups and $C^{*}$-algebras, Ann. of Math. 81 (1965), $38-55$.

[6] F. B. Jones, The aposyndetic decomposition of homogeneous continua, Topology Proc. 8 (1983), 51-54.

[7] J. Krasinkiewicz, On homeomorphisms of the Sierpinski curve, Comment. Math. Prace Mat. 12 (1969), 255-257.

[8] P. Krupski, Recent results on homogeneous curves and ANR's, Topology Proc. 16 (1991), 109-118. 
[9] P. Krupski and J. R. Prajs, Outlet points and homogeneous continua, Trans. Amer. Math. Soc. 318 (1990), 123-141.

[10] T. Maćkowiak, Terminal continua and the homogeneity, Fund. Math. 127 (1987), $177-186$

[11] T. Maćkowiak and E. D. Tymchatyn, Continuous mappings on continua II, Dissertationes Math. (Rozprawy Mat.) 225 (1984).

[12] J. C. Mayer, L. G. Oversteegen and E. D. Tymchatyn, The Menger curve. Characterization and extension of homeomorphisms of non-locally-separating closed subsets, ibid. 252 (1986).

[13] P. Minc and J. T. Rogers, J r., Some new examples of homogeneous curves, Topology Proc. 10 (1985), 347-356.

[14] R. L. Moore, Triodic continua in the plane, Fund. Math. 13 (1929), 261-263.

[15] J. R. Prajs, Openly homogeneous continua having only arcs for proper subcontinua, Topology Appl. 31 (1989), 133-147.

[16] J. T. Rogers, Jr., Decompositions of homogeneous continua, Pacific J. Math. 99 (1982), 137-144.

[17] - An aposyndetic homogeneous curve that is not locally connected, Houston J. Math. 9 (1983), 433-440.

[18] -, Aposyndetic continua as bundle spaces, Trans. Amer. Math. Soc. 283 (1984), 49-55.

[19] - Homogeneous curves that contain arcs, Topology Appl. 21 (1985), 95-101.

[20] - Decompositions of continua over the hyperbolic plane, Trans. Amer. Math. Soc. 310 (1988), 277-291.

[21] G. T. Whyburn, Analytic Topology, Amer. Math. Soc. Colloq. Publ. 28, Providence, R.I., 1942.

[22] - , Topological characterization of the Sierpinski curve, Fund. Math. 45 (1958), 320-324.

MATHEMATICAL INSTITUTE

UNIVERSITY OF WROCŁAW

PL. GRUNWALDZKI $2 / 4$

50-384 WROCŁAW, POLAND

E-mail: KRUPSKI@MATH.UNI.WROC.PL 